\title{
Low-dose Epidermal Growth Factor Receptor (EGFR)- Tyrosine Kinase Inhibition of EGFR Mutation-positive Lung Cancer: Therapeutic Benefits and Associations Between Dosage, Efficacy and Body Surface Area
}

\author{
Ryosuke Hirano ${ }^{1,}$ Junji Uchino $^{1 *}$, Miho Ueno ${ }^{2}$, Masaki Fujita ${ }^{1}$, Kentaro Watanabe $^{1}$
}

\begin{abstract}
A key drug for treatment of EGFR mutation-positive non-small cell lung cancer is epidermal growth factor receptor-tyrosine kinase inhibitor (EGFR-TKI). While the dosage of many general anti-tumor drugs is adjusted according to the patient body surface area, one uniform dose of most TKIs is recommended regardless of body size. In many cases, dose reduction or drug cessation is necessary due to adverse effects. Disease control, however, is frequently still effective, even after dose reduction. In this study, we retrospectively reviewed the characteristics of 26 patients at Fukuoka University Hospital between January 2004 and January 2015 in whom the EGFR-TKI dose was reduced with respect to progression free survival and overall survival. There were 10 and 16 patients in the gefitinib group and the erlotinib group, respectively. The median progression-free survival in the gefitinib group and the erlotinib group was 22.4 months and 14.1 months, respectively, and the median overall survival was 30.5 months and 32.4 months, respectively. After stratification of patients by body surface area, the overall median progression-free survival was significantly more prolonged in the low body surface area $(<1.45 \mathrm{~m} 2)$ group (25.6 months) compared to the high body surface area $(>1.45 \mathrm{~m} 2)$ group $(9.7$ months $)(p=0.0131)$. These results indicate that low-dose EGFR-TKI may sufficiently control disease without side effects in lung cancer patients with a small body size.
\end{abstract}

Keywords: NSCLC - low dose EGFR-TKI - low body surface area

Asian Pac J Cancer Prev, 17 (2), 785-789

\section{Introduction}

Treatment for advanced non-small cell lung cancer (NSCLC) has improved and expanded following advances in chemotherapy using cytotoxic drugs. However, NSCLC remains the leading cause of cancer deaths worldwide (Siegel et al., 2014). Therefore, further development of therapeutic approaches is expected and needed. The ability to detect multiple driver gene mutations in lung cancer resulted in substantial changes in its treatment. Specifically, epidermal growth factor receptor (EGFR) gene mutation was reported in approximately $30 \%$ of Japanese lung cancer patients, the most frequent mutation observed (Roengvoraphoj et al., 2013). Thus, EGFR mutation-positive lung cancer is being actively researched. The results of 2 phase III randomized comparative studies in patients with EGFR mutation-positive NSCLC conducted in Japan (NEJ002, WJTOG3405) showed that the clinical benefit of gefitinib was superior to that of conventional chemotherapies. In both studies, the clinical benefit of gefitinib was compared with chemotherapy as the first-line therapy in patients with EGFR mutationpositive NSCLC. Gefitinib clearly prolonged progressionfree survival (PFS) (hazard ratio $<0.5$ in both studies) (Maemondo et al., 2010; Mitsudomi et al., 2010). Japanese guidelines now stipulate new standard therapy for NSCLC using EGFR-TKI based on the abovementioned results, as well as results of the EURTAC study (erlotinib versus standard chemotherapy as first-line treatment for European patients with advanced EGFR mutation-positive nonsmall-cell lung cancer) conducted in Europe comparing erlotinib with chemotherapy, and the OPTIMAL study (erlotinib versus chemotherapy as first-line treatment for patients with advanced EGFR mutation-positive nonsmall-cell lung cancer) conducted in China (Zhou et al., 2011; Rosell et al., 2012).

Standard doses of gefitinib and erlotinib have been established as $250 \mathrm{mg} /$ day and $150 \mathrm{mg} /$ day, respectively, without adjusting for individual patient characteristics. However, in our clinical practice we often encounter 
patients requiring dose reduction due to liver dysfunction or skin disorder. Some studies suggest persistence of the clinical benefit after dose reduction. For instance, the NEJ002 follow-up study showed prolonged PFS in the dose reduction group compared to those who received a full dose (Satoh et al., 2011), and a prospective observational study of patients with EGFR-mutant NSCLC revealed that gefitinib was less effective in individuals with a high BSA (Kudo et al., 2015). However, it remains unclear whether BSA affects the efficacy of EGFR-TKI monotherapy in patients with EGFR-mutant NSCLC.

Here, we retrospectively examined changes in clinical benefits in 26 NSCLC patients in whom the dose of gefitinib or erlotinib was reduced and the relationship between efficacy, dosage, and BSA.

\section{Materials and Methods}

A total of 95 and 65 patients who were treated with gefitinib and erlotinib at the Department of Respiratory Medicine, Faculty of Medicine, Fukuoka University between January 2004 and January 2015, respectively. We retrospectively examined patients with advanced or recurrent NSCLC who received treatment with EGFRTKI, and in whom the dose was reduced. There were 10 and 16 patients in the gefitinib group and the erlotinib group, respectively. All procedures were performed in accordance with the ethical principles expressed in the 1995 Declaration of Helsinki. The institutional review board of Fukuoka University Hospital approved this retrospective study. PFS, overall survival (OS), and the clinical benefit of EGFR-TKI in patients stratified by BSA were examined. The EGFR mutation status in lung biopsy tissue, bronchial lavage cells, or pleural fluid was assessed using the polymerase chain reaction (PCR) clamp method (Yoshioka et al., 2010).

Statistical analysis was performed using the $\chi^{2}$ test for comparisons between two groups. Survival curves were generated by the Kaplan-Meier method and statistically verified using the log-rank test.

BSA was calculated using the following formula (Dubois and Dubois, 1989):

$\operatorname{BSA}\left(\mathrm{m}^{2}\right)=($ body weight $[\mathrm{kg}]) 0.425 \times($ height $[\mathrm{cm}])$ $0.725 \times 0.007184$

\section{Results}

Table 1 shows the patient characteristics. A total of 26 patients were included in the study. There were 15 males and 11 females, and the ages ranged from 38 to 84 years with a median age of 71 years. The BSA range among patients was 1.10-1.86 (median 1.54), and performance status was $0-1$ in all patients. Mean body weight (range) were $53.0(31.2-72.6)$ years.

There were 12 non-smoking patients and 14 smoking patients. Adenocarcinoma was found in 21 patients, while the histological cancer type was unknown in the other 5 patients. EGFR mutation analyses performed for 14 patients showed exon 19 deletions in 4 patients, exon 21 (L858R) point mutations in 7 patients, and unknown EGFR mutation status in 3 patients. Clinical stages IB,
Table 1. Patient Characteristics and Treatment

\begin{tabular}{lccc}
\hline Parameter & Total & Gefitinib & Erlotinib \\
\hline No. of patients & 26 & 10 & 16 \\
Sex & & & \\
$\quad$ Male & 15 & 3 & 12 \\
$\quad$ Female & 11 & 7 & 4 \\
Smoking status & & & \\
Never smoker & 12 & 7 & 5 \\
Smoker & 14 & 3 & 11 \\
Histology & & & \\
Adenocarcinoma & 21 & 9 & 12 \\
Others & 5 & 1 & 4 \\
Clinical stage & & & \\
IB & 1 & 1 & 0 \\
IIIA & 1 & 0 & 1 \\
IIIB & 3 & 1 & 2 \\
IV & 15 & 4 & 11 \\
Postoperative & 6 & 4 & 2 \\
Type of EGFR mutation & & 1 & 3 \\
Exon 19 deletion & 4 & 2 & 5 \\
L858R & 7 & 1 & 2 \\
Unknown & 3 & 0 & 6 \\
Others & 0 & & \\
Not examined & 12 & & \\
\hline
\end{tabular}

Table 2. Treatment Details

\begin{tabular}{lcc}
\hline & Gefitinib & Erlotinib \\
\hline Given at standard dose & $\mathrm{n}=10$ & $\mathrm{n}=16$ \\
Mean days (SD) & $125(81)$ & $52(45)$ \\
Median days (range) & $90(37-257)$ & $52(0-196)$ \\
Given at low dose & $\mathrm{n}=10$ & $\mathrm{n}=16$ \\
Mean days (SD) & $238(219)$ & $345(429)$ \\
Median days (range) & $181(5-580)$ & $241(2-1496)$ \\
Treatment break period & $\mathrm{n}=3$ & $\mathrm{n}=6$ \\
Mean days (SD) & $65(64)$ & $20(11)$ \\
Median days (range) & $28(12-154)$ & $18(7-40)$ \\
\hline
\end{tabular}

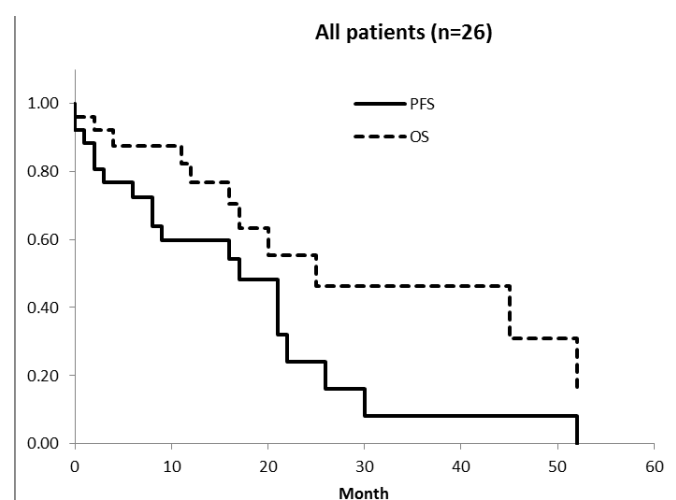

Figure 1. Progression-free Survival and Overall Survival in All Patients

IIIA, IIIB, and IV were found in 1, 1, 3, and 15 patients, respectively, and postoperative recurrence was found in 6 patients. There were 10 and 16 patients in the gefitinib group and the erlotinib group, respectively. Patient characteristics for each drug group are also shown in Table 1.

The dose reductions were as follows: in the gefitinib group, 5 patients received $250 \mathrm{mg}$ every 2 days and the other 5 patients received other regimens; in the erlotinib group, 14 patients received $100 \mathrm{mg}$ daily and the other 2 patients received other regimens. The dosing time periods 


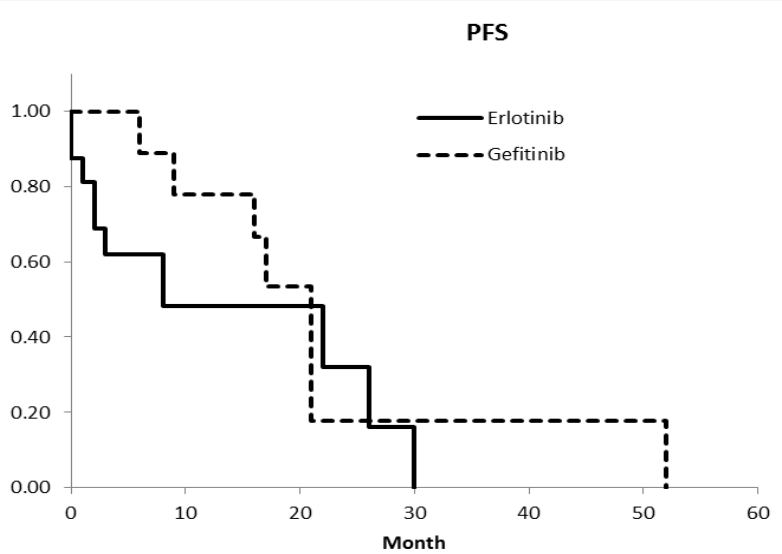

Figure 2. Progression-free Survival in the Gefitinib and Erlotinib Groups

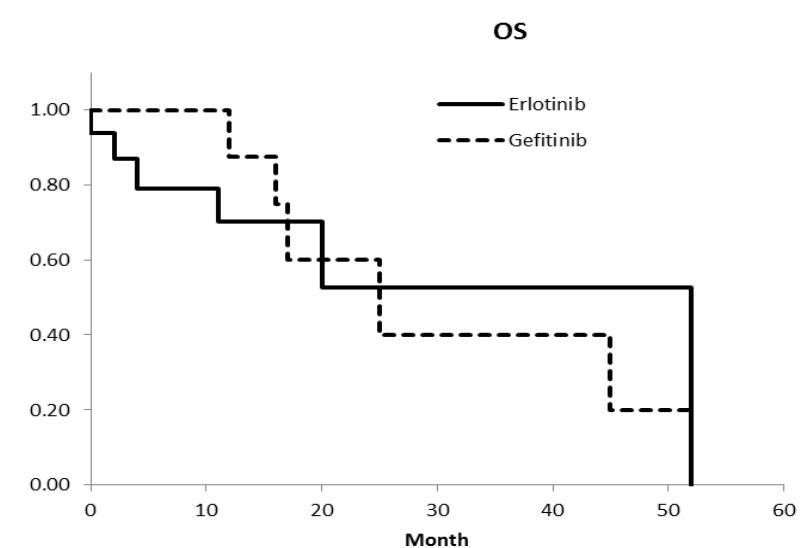

Figure 3. Overall Survival in the Gefitinib and Erlotinib Groups

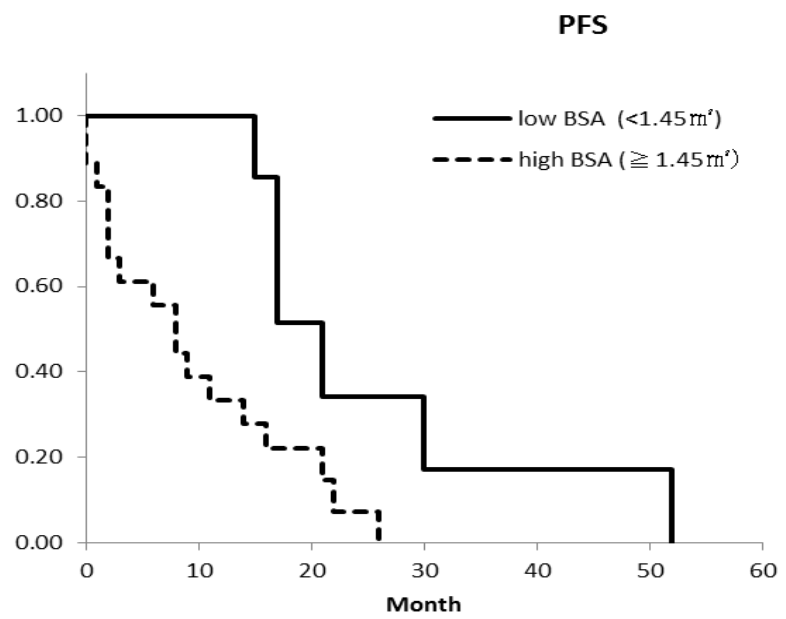

Figure 4. Progression-free Survival in the Low Body Surface Area (BSA $<1.45 \mathrm{~m} 2)$ Group and the High BSA (>1.45 m2) Group

for the recommended doses and the reduced doses are shown in Table 2.

When all patients were considered together, the median PFS was 17.4 months and the median OS was 30.6 months (Figure 1). The median PFS in the gefitinib group and the erlotinib group was 22.4 months and 14.1 months, respectively (Figure 2), and the median OS was 30.5 months and 32.4 months, respectively (Figure 3 ). After stratification of the data according to patients with low $\operatorname{BSA}\left(<1.45 \mathrm{~m}^{2}\right)$ versus high $\mathrm{BSA}\left(>1.45 \mathrm{~m}^{2}\right)$, the overall os

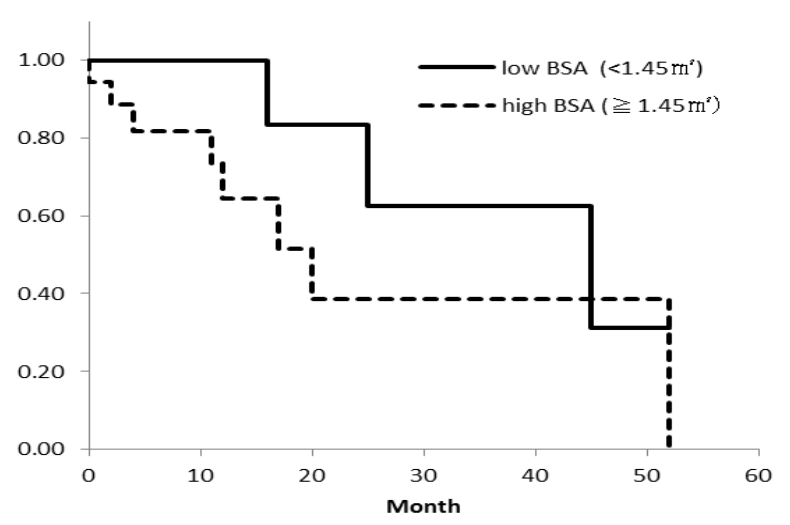

Figure 5. Overall Survival in the Low BSA $(<1.45 \mathrm{~m} 2)$ Group and the High BSA ( $>1.45 \mathrm{~m} 2)$ Group

median PFS was 25.6 months and 9.7 months, respectively (Figure 4). The low BSA group had a statistically significantly prolonged PFS compared to the high BSA group $(\mathrm{p}=0.0131)$. The median OS was 38.2 months and 27.2 months, respectively $(\mathrm{p}=0.189)$ (Figure 5).

\section{Discussion}

In the Phase I study for gefitinib (Nakagawa et al., 2003), patients experienced dose limiting toxicity (DLT) at a dose of $700 \mathrm{mg} /$ day. Based on this result, 2 global phase II clinical studies (Fukuoka et al., 2003; Kris et al., 2003) (IRESSA Dose Evaluation in Advanced Lung Cancer Trial: IDEAL-I and II) were conducted to compare doses of $250 \mathrm{mg} /$ day and $500 \mathrm{mg} /$ day, and the results revealed no differences in clinical benefit between groups. The recommended dose was therefore set at $250 \mathrm{mg} /$ day because adverse effects were more often observed in the $500 \mathrm{mg} /$ day group. That is, in contrast to cytotoxic drugs that have recommended doses determined by conventional DLT studies, the anti-tumor effects of gefitinib seem to plateau at a certain dose, while the incidence of adverse effects increases in a dose-dependent manner. Considering this pharmacological profile, adverse effects may decrease with further gefitinib dose reduction in some patients, while anti-tumor clinical benefits are maintained. The blood concentration of anti-tumor drugs was reported to be closely correlated with their anti-tumor effects (Miles et al., 2010), and the doses of cytotoxic drugs are frequently adjusted according to the BSA of the patient. Low-dose imatinib, a BCR-abl-TKI, was reported to sufficiently control disease in patients with a small BSA (Kawaguchi et al., 2009). Yeo et al. (2010) indicated that low dose gefitinib and erlotinib showed cytotoxic effects in NSCLC cell lines in vitro. Moreover, they reported in a retrospective study that partial remission was obtained in 5 out of the 7 patients who received treatment with 25 $\mathrm{mg}$ /day of erlotinib, since their drug concentrations in serum were considered pharmacologically equal to those in patients who received $250 \mathrm{mg} /$ day of gefitinib. The median PFS for patients on the low dose of erlotinib was 17 months, comparable to the PFS in other reports (Yeo et al., 2010). Kudo et al. reported that gefitinib was less effective in individuals with high BSA than in those with 
low BSA (BSA cut-off around $1.50 \mathrm{~m}^{2}$ ), and a significant difference was observed in the median PFS between groups (Kudo et al., 2015). Ichihara et al. also indicated the efficacy of standard gefitinib doses in prolonging PFS in a low BSA group (Ichihara et al., 2013). However, Sato et al. reported no difference in clinical benefits when segregating the data by BSA and between patients with a TKI dose reduction ( $18.8 \%$ of patients in that study) (Sato et al., 2014). Thus, the benefits of dose reduction based on BSA remain controversial.

After considering the above studies, we set out to retrospectively study the clinical benefits and association with BSA in patients who experienced dose reduction of gefitinib or erlotinib in order to control adverse effects. In this study, no significant correlation was found between OS and BSA in patients in whom the dose was reduced. However, PFS was significantly prolonged in the low BSA group (BSA cut-off of $1.45 \mathrm{~m}^{2}$ ). The median PFS was 25.6 months in the low BSA $\left(<1.45 \mathrm{~m}^{2}\right)$ group, thus indicating favorable results compared with PFS data (9.7 months) in Japanese patients who received erlotinib monotherapy (Seto et al., 2014).

In the current study, several of the patients had unknown EGFR gene mutation status. However, these patients were in both the low and high BSA groups, and the PFS in the low BSA group was prolonged compared to the PFS of patients in previous studies whose EGFR mutation status was known. Thus, low dose TKI appears to be effective in the low BSA population even when the EGFR mutation status is unknown. The median dosing period after dose reduction was as long as 238 and 345 days in the gefitinib group and in the erlotinib group, respectively, indicating that suppression of adverse effects through dose reduction was achievable while still controlling disease (Table 3 ).

The limitations of this study were that it was retrospective, included a small sample size, drug regimens differed from patient to patient, several of the patients had unknown EGFR mutation status, and the study was conducted in a randomly enrolled population. Even so, the results of our study suggest that reduced EGFR-TKI doses decrease adverse effects in patients with a small body size while maintaining PFS, although the risk of disease progression with a reduced dosage may persist. Therefore, a future prospective study investigating the incidence of dose reduction in patients with adverse effects and the clinical benefits in those with low BSA may be useful for clinical practice.

\section{Acknowledgements}

We would like to thank Editage (www.editage.jp) for English language editing.

\section{References}

Dubois D, Dubois EF (1989). Nutrition metabolism classic - a formula to estimate the approximate surface-area if height and weight be known (reprinted from archives internal medicine, Vol 17, Pg 863, 1916). Nutrition, 5, 303-11.

Fukuoka M, Yano S, Giaccone G, et al (2003). Multi-institutional randomized phase II trial of gefitinib for previously treated patients with advanced non-small-cell lung cancer (The IDEAL 1 Trial) [corrected]. J Clin Oncol, 21, 2237-46.

Ichihara E, Hotta K, Takigawa N, et al (2013). Impact of physical size on gefitinib efficacy in patients with non-small cell lung cancer harboring EGFR mutations. Lung Cancer, 81, 435-9.

Kawaguchi T, Hamada A, Hirayama C, et al (2009). Relationship between an effective dose of imatinib, body surface area, and trough drug levels in patients with chronic myeloid leukemia. Int J Hematol, 89, 642-8.

Kris MG, Natale RB, Herbst RS, et al (2003). Efficacy of gefitinib, an inhibitor of the epidermal growth factor receptor tyrosine kinase, in symptomatic patients with non-small cell lung cancer: a randomized trial. JAMA, 290, 2149-58.

Kudo K, Hotta K, Ichihara E, et al (2015). Impact of body surface area on survival in EGFR-mutant non-small cell lung cancer patients treated with gefitinib monotherapy: observational study of the Okayama Lung Cancer Study Group 0703. Cancer Chemother Pharmacol, 76, 251-6.

Maemondo M, Inoue A, Kobayashi K, et al (2010). Gefitinib or chemotherapy for non-small-cell lung cancer with mutated EGFR. $N$ Engl J Med, 362, 2380-8.

Miles DW, Chan A, Dirix LY, et al (2010). Phase III study of bevacizumab plus docetaxel compared with placebo plus docetaxel for the first-line treatment of human epidermal growth factor receptor 2-negative metastatic breast cancer. J Clin Oncol, 28, 3239-47.

Mitsudomi T, Morita S, Yatabe Y, et al (2010). Gefitinib versus cisplatin plus docetaxel in patients with non-small-cell lung cancer harbouring mutations of the epidermal growth factor receptor (WJTOG3405): an open label, randomised phase 3 trial. Lancet Oncol, 11, 121-8.

Nakagawa K, Tamura T, Negoro S, et al (2003). Phase I pharmacokinetic trial of the selective oral epidermal growth factor receptor tyrosine kinase inhibitor gefitinib ('Iressa', ZD1839) in Japanese patients with solid malignant tumors. Ann Oncol, 14, 922-30.

Roengvoraphoj M, Tsongalis GJ, Dragnev KH, et al (2013). Epidermal growth factor receptor tyrosine kinase inhibitors as initial therapy for non-small cell lung cancer: focus on epidermal growth factor receptor mutation testing and mutation-positive patients. Cancer Treat Rev, 39, 839-50.

Rosell R, Carcereny E, Gervais R, et al (2012). Erlotinib versus standard chemotherapy as first-line treatment for European patients with advanced EGFR mutation-positive non-smallcell lung cancer (EURTAC): a multicentre, open-label, randomised phase 3 trial. Lancet Oncol, 13, 239-46.

Sato S, Kurishima K, Miyazaki K, et al (2014). Efficacy of tyrosine kinase inhibitors in non-small-cell lung cancer patients undergoing dose reduction and those with a low body surface area. Mol Clin Oncol, 2, 604-8.

Satoh H, Inoue A, Kobayashi K, et al (2011). Low-dose gefitinib treatment for patients with advanced non-small cell lung cancer harboring sensitive epidermal growth factor receptor mutations. J Thorac Oncol, 6, 1413-7.

Seto T, Kato T, Nishio M, et al (2014). Erlotinib alone or with bevacizumab as first-line therapy in patients with advanced non-squamous non-small-cell lung cancer harbouring EGFR mutations (JO25567): an open-label, randomised, multicentre, phase 2 study. Lancet Oncol, 15, 1236-44.

Siegel R, Ma J, Zou Z, et al (2014). Cancer statistics, 2014. CA Cancer J Clin, 64, 9-29.

Yeo WL, Riely GJ, Yeap BY, et al (2010). Erlotinib at a dose of $25 \mathrm{mg}$ daily for non-small cell lung cancers with EGFR mutations. J Thorac Oncol, 5, 1048-53.

Yoshioka H, Hotta K, Kiura K, et al (2010). A phase II trial of erlotinib monotherapy in pretreated patients with advanced 

non-small cell lung cancer who do not possess active EGFR mutations: Okayama Lung Cancer Study Group trial 0705. J Thorac Oncol, 5, 99-104.

Zhou C, Wu YL, Chen G, et al (2011). Erlotinib versus chemotherapy as first-line treatment for patients with advanced EGFR mutation-positive non-small-cell lung cancer (OPTIMAL, CTONG-0802): a multicentre, openlabel, randomised, phase 3 study. Lancet Oncol, 12, 735-42. 